Perspectiva Geográfica

ISSN 0123-3769

Vol. 15/2010; pp. 277-298

\title{
Perspectiva territorial de la gestión de riesgos de desastres en Colombia
}

Territorial perspective in the risk management of disasters

in Colombia

Martha Teresa Martínez Rubiano*

\section{Resumen}

La prevención y atención de desastres en Colombia se consideraba hasta hace una década como algo novedoso; actualmente, la gestión del riesgo de desastres es proyectada en diferentes dimensiones del desarrollo en correlación con otras políticas del Estado, con el propósito de conjugar iniciativas, propuestas y esfuerzos que garanticen su adecuada ejecución. Sin embargo, hasta la fecha los avances en la gestión del riesgo para reducir los desastres son insuficientes. En Colombia se han implementado diferentes políticas de apoyo a la gestión del riesgo; una de las más importantes es el Ordenamiento Territorial, implementado en cada uno de los municipios con el objetivo principal de proteger a la población en las zonas de amenaza y riesgo. En este artículo se realiza una revisión de la gestión de los riesgos de desastres, para luego presentar la dimensión territorial del desarrollo en Colombia, y la perspectiva territorial de la gestión de riesgos. Para terminar, se realiza un análisis de las aplicaciones de todas estas medidas en la situación de gestión de riesgos en Colombia.

Palabras clave: Desarrollo, Gestión, Ordenamiento, Riesgo, Territorio.

Magíster en Geografía. Docente Universidad del Cauca.mtmartinez@unicauca.edu.co, mtmartinezr@gmail.com 


\begin{abstract}
Prevention and disaster relief in Colombia, up until a decade ago, was considered to be something of a novelty; Currently, disaster risk management is projected on different dimensions of development in correlation with other State policies, with the aim of combining efforts, proposals and initiatives in order to ensure its proper implementation. However, so far advances in the risk management for disaster reduction are insufficient. In Colombia various support policies have been implemented for the management of risk; one of the most important ones is that of zoning implemented in each of the municipalities with the aim of protecting the population in the areas of threat and risk. In this article we make a review of the management of the risks of disasters, to then present the territorial dimension of development in Colombia, and the territorial perspective of risk management. In conclusion an analysis is made of the application of these measures in terms of the situation of risk management in Colombia.
\end{abstract}

Keywords: Development, Management, Risk Management, Territory. 


\section{Introducción}

El crecimiento de la población colombiana, de las infraestructuras y de la producción ha generado la progresiva ocupación de los territorios disponibles, con la particularidad de concentrarse en áreas de la Cordillera Andina o en áreas bajas, expuestas a fenómenos naturales amenazantes. La sociedad, según sus condiciones sociales, culturales y económicas, sufre pérdidas continuas derivadas de la ocurrencia de desastres de diferente magnitud ocasionados por fenómenos naturales, las cuales se acrecientan con el paso del tiempo, limitando las posibilidades de desarrollo.

Los expertos de los riesgos de desastres plantean que aunque es imposible impedir la aparición de los fenómenos naturales amenazantes, como los terremotos, los tsunamis, las erupciones volcánicas y las inundaciones, sí se pueden mitigar sus impactos. Desde este punto de vista, la magnitud de cualquier desastre va íntimamente ligada a la exposición de los territorios a un fenómeno natural amenazante; de igual manera, es necesario conocer la capacidad que tiene una comunidad y su medioambiente para resistir el impacto de la amenaza, como también la capacidad de resiliencia y adaptación de las comunidades y su medioambiente. En este contexto, es necesario hacer converger los procesos de conocimiento de las condiciones locales con proyectos político-administrativos y de participación comunitaria, para generar lo que se ha definido como la gestión del riesgo, que asume la toma de medidas preventivas para la reducción del riesgo y provee las condiciones para una mejor calidad de vida de la población, garantizando la sostenibilidad ambiental; es de esta manera que los esfuerzos deben ser orientados hacia la armonización de los aspectos ambientales, económicos y sociales con el territorio, a través de herramientas como la planificación del desarrollo territorial a diferentes escalas: nacional, regional y local.

Hasta la fecha, los avances en la gestión para reducir los desastres son insuficientes; a nivel mundial se ha firmado el Marco de Acción de Hyogo (Conferencia Mundial sobre la Reducción de los Desastres, 2005), que acuerda que para el año 2015 se realice una reducción considerable de las pérdidas ocasionadas por los desastres; de igual manera, en el tema de cambio climático se concertó, en diciembre de 2009, el "Acuerdo de Copenhague ${ }^{1}$ ", para dar impulso a acciones inmediatas sobre el cambio climático para una mayor seguridad ambiental planetaria y guiar las negociaciones hacia la acción a largo plazo. A partir de estos acuerdos, Colombia ha participado en diferentes proyectos de apoyo a la gestión del riesgo desde el Ministerio de Ambiente, Vivienda y Desarrollo Territorial (MAVDT) y la Dirección de Gestión del

Acuerdo de Copenhague, en: http://copenhagen2009.blogspot.com/ 
Riesgo, entre otras instituciones, con el propósito de realizar la asistencia para establecer programas para la prevención y reducción de riesgos. Unos de los proyectos más importantes para la gestión del riesgo en el país son los Planes de Ordenamiento Territorial desarrollados en cada uno de los municipios de Colombia; una de las temáticas que incorporan es la gestión de riesgos, con el objetivo de proteger a la población en las zonas de amenaza y riesgo.

A partir de este panorama, este artículo se propone realizar una revisión de la gestión de los riesgos de desastres, haciendo énfasis en el ámbito del desarrollo territorial en Colombia. En primer lugar, se presenta un contexto teórico y conceptual del tema de la gestión de riesgos de desastres; en segundo lugar, se presenta la dimensión territorial del desarrollo en Colombia, y por último, la perspectiva territorial de la gestión de riesgos, en la que se destaca su incorporación con el desarrollo territorial, enfatizando en los planes de ordenamiento territorial. Para terminar, se realiza un análisis de los impactos o efectos territoriales de todas estas medidas en la situación de gestión de riesgos en Colombia.

\section{La gestión de riesgos de desastres}

En la década de los noventa del siglo pasado, los estudios sobre los riesgos y los desastres alcanzaron gran interés, gracias a la promoción realizada por las Naciones Unidas; durante esta década se desarrolló un programa mundial para consolidar unos conocimientos sobre riesgos de desastres y establecer las estrategias para reducir el impacto de los fenómenos extremos que causan destrucción, bajo la influencia de diferentes disciplinas académicas e instituciones especializadas; tanto los conceptos como las estrategias reflejaron diferentes perspectivas y enfoques para explicar las condiciones de los riesgos de desastres en el mundo.

En este artículo nos centraremos en uno de los enfoques más relacionados con el tema de la gestión del riesgo, como es el desarrollado a través del trabajo científico basado en experiencias, análisis y explicaciones, que lleva a producir políticas, procedimientos, experimentos y normas. Las ciencias de la tierra y las ciencias aplicadas son las que se enmarcan en este enfoque, ellas han brindado los conocimientos que permiten al ser humano tratar de controlar la naturaleza y satisfacer así las necesidades de su existencia. Estas disciplinas se complementan con una tendencia reciente, denominada ciencias sistémicas y de la complejidad, que busca el conocimiento integral con preferencia de estudios a escalas locales.

Los aportes teóricos y metodológicos de las ciencias de la tierra se concentraron, inicialmente, en la conceptualización y el análisis de los desastres, y fueron asumidos por los especialistas con investigaciones dirigidas a conocer fenómenos geodinámicos, como terremotos, erupciones volcánicas y 
deslizamientos, y fenómenos hidrometeorológicos, como huracanes e inundaciones, entre otros. Originariamente, esta tendencia identificó estos eventos físicos extremos como "desastres naturales", es decir, el evento natural era asumido por sí mismo como un suceso de efectos negativos, sin considerar la ocupación del territorio (Calderón, 2001).

En las investigaciones de los desastres bajo este enfoque, se estudian las características, el funcionamiento y la probabilidad de ocurrencia de los fenómenos en un lugar y un tiempo dados con metodologías positivistas propias de cada ciencia. El propósito es conocer los fenómenos relacionados como amenazas, concepto originado en este tipo de ciencias. Al determinar aspectos de fenómenos naturales que causan los desastres, el propósito es lograr su predicción exacta. Las amenazas han sido definidas como eventos físicos, potencialmente peligrosos, los cuales pueden tener diferentes orígenes: natural (geológico, hidrometeorológico, biológico) o antrópico (degradación ambiental, accidentes de transporte, contaminación industrial, destrucción de estructuras, incendios, accidentes tecnológicos) (Cardona et al., 2001, 2004). En este artículo consideramos la gestión del riesgo de desastres a partir de las amenazas de origen natural.

Desde los años sesenta, las ciencias aplicadas, como las ingenierías, la arquitectura y la economía, han realizado múltiples estudios, ampliando sus conocimientos en relación con los efectos de los desastres, en cuanto a pérdidas y daños; de ellos surge el concepto de magnitud de un desastre o del riesgo, no solo en función del evento natural, sino de las pérdidas físicas, de los daños causados y sus costos. Las disciplinas incluyeron en los estudios la cuantificación de los impactos de una amenaza tanto a nivel físico como ambiental y socioeconómico, entre otros; de igual manera, se enfocaron al estudio del impacto particular de eventos asociados con amenazas de diferente tipo: en el territorio, en las infraestructuras, en la economía y en los sistemas sociales, entre otros, introduciendo un concepto clave, el de vulnerabilidad, asumido como las condiciones determinadas de factores o procesos que aumentan la susceptibilidad de una comunidad al impacto de amenazas (Blaikie et al., 1997). Los estudios de vulnerabilidad han analizado algunos principios, planteados por Ratick, como:

Exposición: Derivada de la intersección de la actividad humana, el uso del suelo y el medio ambiente construido, con los patrones de amenaza.

Resistencia: Es la capacidad que tiene una sociedad y el medioambiente para resistir el impacto de los eventos amenazantes.

Resiliencia: Es la capacidad de una sociedad para recuperarse de un desastre.

Aprendizaje: Considerado como la capacidad de una sociedad de aprender de los desastres ocurridos. 
Adaptación: Es la capacidad de una sociedad para cambiar sus patrones de conducta a raíz de la ocurrencia del desastre (Ratick, citado en Maskrey, 1989).

Estos principios permiten conocer las diferentes potencialidades de la comunidad para prepararse, soportar y reacomodarse a los posibles impactos generados por el evento; aquí es importante evaluar las condiciones históricas para conocer cómo han evolucionado algunos componentes dentro de la vulnerabilidad, y de esta forma comprender la situación en la que vive la comunidad (Martínez, 2005; GIRA, 2003). La vulnerabilidad, como factor diferencial, debe ser diagnosticada según cada amenaza, como terremoto, tsunami, licuación o inundación, con sus eventos asociados. Además, el análisis no puede estar dirigido únicamente a la pérdida o daño de la vida misma y de los bienes, sino de los desequilibrios o impactos en los medios de subsistencia que componen las relaciones diarias de una sociedad.

Estudios como los realizados por Chías (2004, 2009), en términos de accidentalidad vial, podrían brindarnos algunos modelos de manejo de variables en términos territoriales. La vulnerabilidad no se encuentra solamente en factores como la pobreza, se deben tener en cuenta características como incapacidad, edad y organización, así como las condiciones políticas, económicas e institucionales del medio en el que se desarrolla la comunidad. Se ha subrayado también que los impactos relacionados con las amenazas demuestran grandes diferencias según el territorio y las condiciones sociales en función de la época y fase de desarrollo en la que se encuentren.

Estudiando las amenazas y la vulnerabilidad, como dos fundamentos de una dinámica o un proceso, se puede, además de elaborar el diagnóstico, establecer los riesgos. En la literatura especializada, el riesgo existe porque se presenta una interacción y relación dinámica y compleja entre factores de amenaza y factores de vulnerabilidad, en espacios o territorios definidos (Lavell, 2003; Cardona et al., 2001, 2004). En otros términos, los riesgos son definidos como el daño o las pérdidas posibles en los elementos expuestos como consecuencia de las condiciones de vulnerabilidad de estos frente a las amenazas que los afectan (Thomas, 2006).

Los riesgos pueden caracterizarse desde diferentes dimensiones (GIRA, 2003; Martínez, 2007):

- Dimensión espacial: El riesgo de desastre se manifiesta en territorios definidos y circunscritos, y es sufrido por individuos, familias, colectividades humanas, sistemas productivos o infraestructuras ubicados en sitios determinados. Los desastres tienen una expresión territorial definida que varía desde lo muy local hasta cubrir vastas extensiones; la expresión más fehaciente del riesgo se encuentra en los niveles microterritoriales. 
- Dimensión temporal: El riesgo tiene unas causas reconocibles, es un proceso que se construye en el tiempo y se caracteriza por ser dinámico y cambiante. El riesgo es la condición previa al desastre.

- Dimensión social: El riesgo lo han caracterizado como una construcción social, donde las amenazas juegan su parte pero no definen el problema por sí mismas, como sí lo hace la sociedad, la cual es diferenciada por las condiciones sociales, económicas, culturales y psicosociales.

- Dimensión de desarrollo o integral: Según Lavell (2003), los riesgos de desastres se relacionan de una u otra forma con una suma de prácticas humanas inadecuadas que indican una relación desequilibrada con el ambiente que ocupan las sociedades y que son, a la vez, representaciones del déficit en el desarrollo. Una solución del problema de riesgo de desastre es un proceso sujeto a los esquemas de planificación del desarrollo territorial, sectorial y ambiental. Con este reconocimiento, necesariamente la manera en que se considera la intervención humana a favor de manejar el problema, cambia de forma importante.

Estas dimensiones se deben tener en cuenta cuando se presenta la oportunidad de actuar adecuadamente sobre el riesgo, con el fin de evitarlo o mitigarlo, lo que se define como Gestión del Riesgo; los expertos de la RED ${ }^{2}$ lo definen como la capacidad de las sociedades y de sus actores sociales para conocer y transformar el riesgo, actuando sobre las causas que los producen; comprende medidas y formas para reducir, mitigar, prevenir y atender los desastres (Lavell, 2003). Otros autores, como la OPS y Thomas (2006), complementan la definición incluyendo en esta gestión un proceso de planificación, organización, dirección, ejecución y control, dirigido al conocimiento de amenazas y las condiciones de vulnerabilidad frente a ellas, con el propósito de formular e implementar acciones para la reducción de riesgos, el manejo de desastres y la recuperación ante eventos ocurridos.

Algunos de los principios formulados en la gestión de riesgos están orientados a argumentar cómo los desastres son la materialización de riesgos no manejados y relacionados directamente con las condiciones de vulnerabilidad, las cuales deben ser identificadas, analizadas y gestionadas hacia la mitigación o reducción de la probabilidad de un futuro desastre (Lavell, 1994, 1998). Otro argumento está dirigido hacia la comprensión de la importancia de la participación de los actores sociales, que están inmersos en unas relaciones que han creado las condiciones de riesgo y deben participar en su reducción y manejo como un punto de partida para una adecuada gestión del riesgo (Thomas, 2006).

Red de Estudios Sociales en Prevención de Desastres en América Latina. 2009. Desimventar (Boletín informativo y documento consecutivo en línea) Versión 5.4. Cali, Colombia: Observatorio Sismológico del Sur Occidente. Universidad del Valle. 
El enfoque predominante en la gestión de riesgos ha cambiado en las últimas décadas; anteriormente, las acciones y formas de intervención se dirigían al desastre mismo, a la pérdida humana, material, económica y ambiental en un territorio, la cual excede la capacidad de la sociedad afectada para enfrentarla utilizando sus propios recursos, y que probablemente volvería a ocurrir (Cuny, 1983); desde este enfoque se priorizan las acciones de atención a las emergencias y a los preparativos para enfrentar los desastres (DNP, 2001; MAVDT, 2006). Actualmente, al aumentar los desastres y encontrar muchos territorios en permanente situación de riesgo, las acciones y formas de intervención deben orientarse a la transformación de aquellas condiciones o factores de riesgo que de no ser corregidos desencadenan un desastre (MAVDTSNPAD-DNP, 2005, 2006).

Con respecto al primer enfoque, que predominó en Colombia durante las últimas décadas del siglo $\mathrm{xx}$, se definía política y administrativamente como "Prevención y Atención de Desastres", y tiene su origen en Colombia en 1979, cuando se crea el Comité Nacional de Emergencias, mediante la Ley 9 del 79, en la que aparece la primera reglamentación sobre el manejo de los desastres. Durante esos años se presentaron tres desastres de gran magnitud: el tsunami de Tumaco, en 1979; el terremoto de Popayán, en 1983, y la avalancha de Armero, en 1985; hechos que impulsaron cambios políticos y socio-económicos para la búsqueda de soluciones, como la creación en 1988 de un Sistema Nacional para la Prevención y Atención de Desastres (Ley 46), sistema que estructurara las acciones no solamente de atención a las emergencias, sino de prevención a los desastres, conceptos más amplios a los manejados hasta el momento.

En 1989 se expidió el Decreto Ley 919 y se creó la Oficina Nacional para la Prevención y Atención de Desastres, como respuesta a la urgente necesidad que tenía el Gobierno Nacional de afrontar mediante una organización adecuada los innumerables problemas sociales y económicos generados por los distintos desastres ambientales y antrópicos que durante las últimas décadas del siglo xx había sufrido la sociedad colombiana. Una década después se logra la institucionalización de los riesgos de desastres; según el Decreto 93 de 1998, se adopta el Plan Nacional de Prevención y Atención de Desastres, PNPAD, que define las principales acciones en el campo de la gestión de riesgos, enmarcadas en tres objetivos básicos: reducción de riesgos y prevención de desastres, respuesta efectiva en caso de desastres y recuperación rápida de zonas afectadas; el Plan es un marco político integral del tema en relación con el desarrollo y su planificación.

Desde el punto de vista político, la prevención y atención de desastres en Colombia se consideraba hasta hace una década como algo novedoso; actualmente es proyectada en diferentes dimensiones del desarrollo y en correlación con otras políticas del Estado, con el propósito de 
conjugar iniciativas, propuestas y esfuerzos para una adecuada gestión del riesgos en beneficio del desarrollo sostenible que permita generar condiciones favorables para orientar los territorios; de esta manera, en el país se ha acudido a la política de Desarrollo Territorial, que implementa a nivel municipal los Planes de Ordenamiento Territorial. Hoy una serie de ciudades y municipios ejecutan la gestión del riesgo de forma apropiada, conjuntamente con los planes de desarrollo y el ordenamiento territorial, aunque estas situaciones no pueden considerarse generalizadas en el país.

\section{Dimensión territorial del desarrollo}

La dimensión territorial se basa en conceptos como territorio y territorialidad. Para Farinós y Romero (2007), el territorio es un espacio delimitado y atribuido a un grupo humano o una sociedad, donde vive y al que da forma en un proceso de ocupación, asignándole valores según sus rasgos culturales, e incluso simbolismos de identidad y de orden espiritual. Otros autores argumentan, de igual manera, que el territorio forma parte de la construcción social del espacio que cada sociedad organiza según la espacialidad que le es propia y que depende de sus expresiones y de sus normas, así como también de la elección de sus actividades y de su dominio técnico. Existen otros enfoques que interpretan el territorio según sus aspectos físicos o como parte de un desarrollo histórico de la relación sociedad-naturaleza, ya sea para ver el territorio como recurso o como un proceso de artificialización del medioambiente determinado (Bertrand, 1988).

La territorialidad se define como la relación dinámica entre los componentes sociales, como la economía, la cultura, las instituciones y los poderes políticos, $\mathrm{y}$ aquello que es propio del territorio donde se habita, se vive y se produce (Farinós y Romero, 2007); otra concepción de territorialidad la ve como un componente geográfico clave para comprender cómo la sociedad y el espacio están íntimamente relacionados. Desde la perspectiva política es la adscripción de una sociedad o persona a un determinado espacio delimitado, donde adquiere derechos y deberes, y en el que rige un determinado gobierno y ordenamiento. Desde este punto de vista, las políticas de ordenamiento territorial crean territorialidad al determinar las condiciones vigentes dentro de un espacio. Estas conceptualizaciones son establecidas como complementarias, si se consideran dimensiones como ordenamiento y desarrollo de un espacio.

La condición de orden, o de disponer las cosas en un espacio, forma parte de una tendencia científica y de procesos sociales predominantes en algunas culturas. La ordenación es relativa a los objetivos, los criterios y las condiciones del territorio; el orden se hace más complejo y difícil conforme se amplían los elementos y factores que intervienen en el espacio por ordenar. En un sentido básico, la ordenación está relacionada con determinadas prácticas políticas, se 
refiere a la localización, delimitación y situación (relativa) de los hechos presentes en el espacio, principalmente de aquellos elementos a los que se les atribuyen funciones básicas, estructurales, estratégicas o con mayores repercusiones en un orden o modelo territorial (Farinós y Romero, 2007). La ordenación del territorio, como política, implica actuar sobre las actividades que se realizan en él, para fortalecerlas o desestimarlas, cambiarlas, reubicarlas y orientar su comportamiento futuro (Massiris, 2000). Al respecto, algunos autores, como Farinós y Romero (2007), argumentan que en la práctica esta política no puede abarcar todas las actividades del territorio; un plan de ordenación debe expresar los hechos a los que se les atribuye valor deseado, pero no puede ser un instrumento de previsión y control de todos los hechos presentes en el territorio, ni de todos sus factores.

De manera complementaria, la dimensión de desarrollo territorial sostenible es planteada por Massiris (2009), en un ideal latinoamericano, como una convergencia de los objetivos de desarrollo económico con los de desarrollo social, del aprovechamiento sostenible de los recursos naturales con la protección de las condiciones ambientales, como la gestión de riesgos que posibilite el desarrollo integral y sostenido, enmarcado por factores como la descentralización, la democracia, la gobernabilidad y, sobre todo, el territorio, que es la base de todo el desarrollo. Al respecto, Santos (2000) interpreta el territorio, en el contexto del desarrollo, como un todo que se convierte en un elemento de esa armonía forzada entre lugares y la economía, la sociedad y el ambiente allí situados, en función de un poder político mayor situado en centros motores de la administración, es decir, donde la descentralización, la democracia y la gobernabilidad son el propósito; sin embargo, aún no son reales. Sería importante examinar las políticas y realidades del desarrollo territorial en países como Colombia para comprender su evolución en el camino de este proceso.

Con relación a lo anterior es pertinente tener en cuenta los planteamientos de Hildenbrand (2007), quien desde una concepción europea propone que el desarrollo territorial debe cumplir tres funciones: la de ordenación, la de desarrollo y la de coordinación. La primera es propia de espacios sometidos a fuertes presiones en los usos del suelo, entre ellos la extracción de recursos naturales, de las que se derivan impactos negativos que exigen la proyección de un modelo sostenible; esta es una función más preventiva. La función de desarrollo es de carácter más potencial, y contempla relaciones multiescalares; es propia tanto de espacios menos desarrollados, que pueden aprovechar su capital territorial disponible, como de espacios más desarrollados, para reforzar su papel como motores de desarrollo. La tercera función, la de coordinación, se refiere a la forma como se produce esa política pública dirigida a la planificación integral y transectorial del territorio; se relaciona directamente con lo que se ha definido como 
gobernanza territorial a diferentes niveles, escalas y de organizaciones sociales.

A partir de estos planteamientos de ordenamiento y desarrollo se puede revisar cómo se manifiesta la dimensión territorial en Colombia. Los antecedentes de las políticas territoriales se remontan a las últimas décadas del siglo $\mathrm{xx}$, pues antes se advierte un profundo debilitamiento de la actuación pública en términos territoriales, donde predominaban las intervenciones sectoriales y privadas poco coordinadas y en algunos casos indiferentes al establecimiento de un equilibrio del desarrollo territorial. Durante la década de los setenta se implementaron los primeros instrumentos de desarrollo municipal, denominados Planes Integrales de Desarrollo, que deberían estar coordinados con el Plan Nacional de Desarrollo; sin tener unas condiciones favorables, el único logro que se tuvo fue el aislamiento de la planeación urbana-territorial de los planes de desarrollo económico-sociales.

En términos de planeación, en Colombia los años ochenta y noventa significaron una etapa de progreso, con cambios muy importantes en la relación de los entes territoriales con el gobierno nacional, soportados en el inicio de un proceso de descentralización administrativa, la creación de los Consejos Regionales de Planeación Económica y Social y la promulgación de una nueva constitución en 1991, que incluyó leyes reglamentarias sobre temas de desarrollo y ordenamiento territorial. La nueva constitución destaca tres principios que tienen que ver directamente con el desarrollo territorial: la función social y ecológica de la propiedad, el predominio del interés general sobre el particular y la distribución de presupuestos para el desarrollo de los municipios.

Tras la expedición de la constitución de 1991 se sancionó una ley de Ordenamiento Territorial en Colombia, la ley 388 de 1997 (MAVDT-ESAP-ONU, 2004), que tuvo un proceso de discusión y conformación de más de diez años, durante los cuales se retomaron enfoques como el desarrollo económico regional y la planeación urbana; de igual manera, retomó en su fase legislativa intereses de diversas orientaciones políticas, hasta llegar a crear un instrumento para el desarrollo físico, social y económico, encauzado en gran medida a los espacios urbanos, y en menor importancia a los espacios rurales, aspecto que se refleja en la baja gobernabilidad en estos territorios, en los que las condiciones de abandono y conflicto se evidencian una década después.

Los objetivos de la ley de Ordenamiento Territorial cumplen con las tres funciones planteadas por Hildenbrand (2007): la ordenación de los territorios municipales en armonía con el desarrollo regional y rural; el desarrollo, al identificar las potencialidades del municipio y plantear las acciones prioritarias, así como lograr una resolución de los conflictos derivados de la propiedad, la tenencia y el uso del suelo, además de garantizar a los ciudadanos los beneficios del desarrollo territorial como urbanización, servicios, 
espacio público y áreas de protección; y la coordinación, que se plantea al promocionar el trabajo integrado de las entidades estatales de orden nacional, regional y municipal en términos de programas, planes y proyectos, facilitando su ejecución.

El proyecto inicial del ordenamiento territorial en Colombia se promulgó como un proceso en el que en una primera etapa se trabajaba en los municipios, y posteriormente se avanzaba a la planeación territorial regional y nacional con enfoques multiniveles e integrales. Las condiciones políticas del país no permitieron la continuidad del proceso, y se priorizaron otros aspectos de la vida nacional, pero se espera que en algún momento se reactiven los procesos de planeación territorial, como es el caso del proyecto de las regiones funcionales o estratégicas integradas con los planes municipales. En el momento se cuenta con el $98,8 \%$ de los municipios colombianos con un plan de ordenamiento territorial acorde con las escalas promovidas según la población de cada uno, diferenciados en complejidad, profundidad del diagnóstico y en la formulación del ordenamiento, siendo el más exigente el plan para los municipios de más de cien mil personas (MAVDT, 2009).

La ley de Ordenamiento Territorial en Colombia tuvo en su comienzo un enfoque contemporáneo, proponiendo el cubrimiento de todo tipo de temáticas, como la integración regional, el predominio del medioambiente y los recursos naturales, la protección de la población en zonas de riesgo, el énfasis en la vivienda y la gestión del suelo, la renovación urbana, la protección al patrimonio urbanístico, el diseño integral de ciudades y la complementariedad entre el desarrollo territorial y el desarrollo económico y social. Estas temáticas trataron de ser aplicadas por los profesionales, pero ante la escasa experiencia y los requerimientos técnicos, presupuestales y legales no fueron cabalmente implementadas en esta primera etapa del ordenamiento territorial.

Una década después de iniciar el proceso de formulación del ordenamiento en los municipios colombianos, el Ministerio de Vivienda, Ambiente y Desarrollo Territorial empieza una nueva etapa con un proyecto de asistencia para la revisión y ajuste de su contenido y aplicación a través de un instrumento denominado Expedientes Municipales ${ }^{3}$, instrumento de evaluación y control muy similar al presentado por Farinós y Romero (2007), en el que se examina la coherencia, pertinencia y ejecución de los planes de ordenamiento territorial y se sugieren proyectos de complementación. Estos instrumentos deben ser aplicados en cada periodo constitucional (4 años).

La política de desarrollo territorial en Colombia ha planteado funciones de ordenación con criterios de

3 Ministerio de Ambiente, Vivienda y Desarrollo Territorial, Viceministerio de Vivienda y Desarrollo Territorial, Subdirección de Asistencia Técnica a Municipios y Regiones. Fuente: www.minambiente.gov.co 
sostenibilidad, equilibrio territorial y con cohesión institucional, a través de una formulación de planes y proyectos relacionados con unas condiciones locales estudiadas y analizadas en diagnósticos específicos concertados con la comunidad; sin embargo, este es un primer paso de gran importancia para el desarrollo del país, y es necesario dar continuidad a su implementación con un proceso de cambio en el orden territorial.

\section{Dimensión territorial de la Gestión de Riesgos}

La dimensión territorial de la gestión de riesgos presentada en este artículo comprende la incorporación de la gestión de riesgos de desastre en la planificación, como una estrategia de desarrollo en Colombia (DNP, 2001). En la década de los noventa se emitieron normas de gran importancia nacional que vinculan el tema de prevención de riesgos y atención de desastres con el medioambiente y el desarrollo del país. La más importante es la Constitución Política de Colombia de 1991, que platea como fines esenciales del Estado "proteger a todas las personas residentes en Colombia, en su vida, honra, bienes, creencias y demás derechos y libertades, y asegurar el cumplimiento de los deberes sociales del Estado y de los particulares. En cuanto al manejo de los recursos naturales, se reglamentaron disposiciones sobre la vivienda digna y el manejo de los recursos naturales, para garantizar el desarrollo sostenible (prevenir y controlar los factores de deterioro ambiental). Como primer paso para la
Planeación, definió la obligatoriedad para las Entidades Territoriales de elaborar su Plan de Desarrollo. Se dispuso, además, en un esquema de descentralización, que el Estado delegaría en las entidades locales la responsabilidad de conocer y atender en forma integral los recursos naturales, y tomar acciones sobre su manejo" (MAVDT, SNPAD, DNP y Acción Social, 2005: 10).

En 1989 se emitió la Ley $9^{a}$, que incluye el tema de la prevención y la reducción de riesgos en la Planificación del Desarrollo Territorial; esta ley dispone la obligatoriedad de incluir en los Planes de Desarrollo acciones concretas para la intervención del territorio, y la definición de responsabilidades y competencias con respecto a la visión del futuro de los municipios (MAVDT, 2005). La Ley 02 de 1991 estableció que los municipios deben tener actualizados los inventarios de las zonas que presenten altos riesgos para la localización de asentamientos humanos. En 1994, ante las dificultades presentadas al incorporar el tema de prevención de riesgos en los planes de desarrollo, se establecen los procedimientos y mecanismos para la elaboración, aprobación, ejecución, seguimiento, evaluación y control de los planes de desarrollo, tanto de la Nación y de las entidades territoriales en la ley 152 o Ley Orgánica del Plan de Desarrollo.

Otra ley de importancia nacional fue la 99 de 1993 o Ley del Medio Ambiente, en la que se establece que la prevención de desastres será materia de interés colectivo, y que las medidas tomadas para 
evitar o mitigar los efectos de su ocurrencia serán de obligatorio cumplimiento. Según esta ley, corresponde a las Corporaciones Autónomas Regionales: ejercer la función de máxima autoridad ambiental en el área de su jurisdicción; participar en los procesos de planificación y ordenamiento territorial para que el factor ambiental se tenga en cuenta en las decisiones que se adopten; realizar actividades de análisis, seguimiento, prevención y control de desastres, y apoyar en los aspectos medioambientales, como la prevención y atención de emergencias y desastres.

En 1997 se presenta la norma que integraría diferentes dimensiones del desarrollo del país; es la Ley 388 de 1997 o Ley de Ordenamiento Territorial, que obliga a los municipios a formular los Planes de Ordenamiento Territorial, e indica que deben contener, entre otros aspectos, las determinantes y componentes relacionadas con el tema de riesgos. En 1998, según el Decreto 879, se reglamentan las disposiciones referentes al ordenamiento del territorio municipal y distrital y a los Planes de Ordenamiento Territorial. Se tendrán en cuenta las prioridades del Plan de Desarrollo del municipio y los determinantes establecidos en normas de superior jerarquía, entre las que se encuentra: las relacionadas con la conservación y protección del medioambiente, los recursos naturales y la prevención de amenazas y riesgos naturales.

Debido a situaciones presentadas en algunos municipios, en el 2004 se promulgó la Ley 4002, por la cual se establece que teniendo en cuenta razones excepcionales, como la declaratoria de desastre o calamidad pública, y por los resultados de estudios técnicos detallados sobre amenazas, riesgos y vulnerabilidad, el alcalde municipal podrá iniciar el proceso de revisión del plan que justifique la recalificación de áreas de riesgo no mitigable y otras condiciones de restricción diferentes a las inicialmente adoptadas en el POT.

Revisando la Ley de Ordenamiento Territorial (Ley 388 de 1997) se han seleccionado los artículos concordantes con la incorporación de la Prevención y Reducción de Riesgos, los cuales son:

- Capítulo I. Artículo 1. ${ }^{\circ}$. Objetivos. "2. El establecimiento de los mecanismos que permitan al municipio, en ejercicio de su autonomía, promover el ordenamiento de su territorio, el uso equitativo y racional del suelo, la preservación y defensa del patrimonio ecológico y cultural localizado en su ámbito territorial y la prevención de desastres en asentamientos de alto riesgo, así como la ejecución de acciones urbanísticas eficientes". "3. Garantizar que la utilización del suelo por parte de sus propietarios se ajuste a la función social de la propiedad y permita hacer efectivos los derechos constitucionales a la vivienda y a los servicios públicos domiciliarios, y velar por la protección del medio ambiente y la prevención de desastres".

- Capítulo I. Artículo 3. ${ }^{\circ}$. Función del urbanismo. "4. Mejorar la seguridad de 
los asentamientos humanos ante los riesgos naturales".

- Capítulo II. Artículo 8. ${ }^{\circ}$. Acción urbanística. "5. Determinar las zonas no urbanizables que presenten riesgos para la localización de asentamientos humanos, por amenazas naturales, o que de otra forma presenten condiciones insalubres para la vivienda". "11. Localizar las áreas críticas de recuperación y control para la prevención de desastres, así como las áreas con fines de conservación y recuperación paisajística".

- Capítulo III. Artículo 10. Determinantes de los POT. "1. Las relacionadas con la conservación y protección del medio ambiente, los recursos naturales y la prevención de amenazas y riesgos naturales. d) Las políticas, directrices y regulaciones sobre prevención de amenazas y riesgos naturales, el señalamiento y localización de las áreas de riesgo para asentamientos humanos, así como las estrategias de manejo de zonas expuestas a amenazas y riesgos naturales".

- Capítulo III. Artículo 12. Componente General del Plan de Ordenamiento. "2.3 La determinación y ubicación en planos de las zonas que presenten alto riesgo para la localización de asentamientos, por amenazas o riesgos naturales o por condiciones de insalubridad".

- Capítulo III. Artículo 13. Componente urbano del Plan de Ordenamiento. "3. La delimitación, en suelo urbano y de expansión urbana, de las áreas de conservación y protección de los recursos naturales, paisajísticos y de conjuntos urbanos, históricos y culturales, de conformidad con la legislación general aplicable a cada caso y las normas específicas que los complementan en la presente ley; así como de las áreas expuestas a amenazas y riesgos naturales". "5. La estrategia de mediano plazo para el desarrollo de programas de vivienda de interés social...; así como los mecanismos para la reubicación de los asentamientos humanos localizados en zonas de alto riesgo para la salud e integridad de sus habitantes, incluyendo la estrategia para su transformación para evitar su nueva ocupación".

- Capítulo III. Artículo 14. Componente rural del Plan de Ordenamiento. "3. La delimitación de las áreas de conservación y protección de los recursos naturales, paisajísticos, geográficos y ambientales, incluyendo las áreas de amenazas y riesgos, o que formen parte de los sistemas de provisión de los servicios públicos domiciliarios o de disposición final de desechos sólidos o líquidos".

- Capítulo III. Artículo 15. Normas urbanísticas. "1.5 Las que definan las áreas de protección y conservación de los recursos naturales y paisajísticos, las que delimitan zonas de riesgo y en general, todas las que conciernen al medio ambiente, las cuales en ningún caso, salvo en el de la revisión del plan, serán objeto de modificación”. "3.2 La localización de terrenos cuyo uso es el de vivienda de interés social y la 
reubicación de asentamientos humanos localizados en zonas de alto riesgo".

- Capítulo III. Artículo 16. Contenido de los Planes Básicos de Ordenamiento. "1.6 $\mathrm{El}$ inventario de las zonas que presenten alto riesgo para la localización de asentamientos humanos, por amenazas naturales o por condiciones de insalubridad". "2.2 La delimitación de las áreas de conservación y protección de los recursos naturales, ...de conformidad con la legislación general aplicable a cada caso y las normas urbanísticas que los complementan, así como de las áreas expuestas a amenazas y riesgos naturales". "2.3 La estrategia de mediano plazo para el desarrollo de programas de vivienda de interés social, incluyendo... los mecanismos para la reubicación de los asentamientos localizados en zonas de alto riesgo para la salud e integridad de sus habitantes, incluyendo lo relacionado con la transformación de las zonas reubicadas para evitar su nueva ocupación".

- Capítulo IV. Artículo 35. Suelo de protección. "Constituido por las zonas y áreas de terrenos localizados dentro de cualquiera de las anteriores clases, que por sus características geográficas, paisajísticas o ambientales, o por formar parte de las zonas de utilidad pública para la ubicación de infraestructuras para la provisión de servicios públicos domiciliarios o de las áreas de amenazas y riesgo no mitigable para la localización de asentamientos humanos, tiene restringida la posibilidad de urbanizarse".
- Capítulo VII. Artículo 58. Motivos de utilidad pública. "b) Desarrollo de proyectos de vivienda de interés social, incluyendo los de legalización de títulos en urbanizaciones de hecho o ilegales diferentes a las contempladas en el artículo 53 de la Ley $9^{a}$ de 1989, la rehabilitación de inquilinatos y la reubicación de asentamientos humanos ubicados en sectores de alto riesgo". "m) El traslado de poblaciones por riesgos físicos inminentes".

- Capítulo XI. Artículo 104. Sanciones urbanísticas. "1... Si la construcción, urbanización o parcelación en terrenos de protección ambiental, o localizados en zonas calificadas como de riesgo...".

- Capítulo XIII. Artículo 121. "Las áreas catalogadas como de riesgo no recuperable que hayan sido desalojadas a través de planes o proyectos de reubicación de asentimientos humanos, serán entregadas a las Corporaciones Autónomas Regionales o a la autoridad ambiental para su manejo y cuidado de forma tal que se evite una nueva ocupación. En todo caso el alcalde municipal o distrital respectivo será responsable de evitar que tales áreas se vuelvan a ocupar con viviendas y responderá por este hecho".

Esta política de ordenamiento territorial en Colombia ha incorporado funciones de gestión de riesgos con criterios de sostenibilidad, equilibrio territorial y con cohesión institucional, a través de una formulación de planes y proyectos 
relacionados con unas condiciones locales estudiadas y analizadas en diagnósticos específicos, formulación detallada y concertados con la comunidad. Aunque en cuestiones de gestión de riesgos son pocos los municipios que han alcanzado los objetivos propuestos, pues en la práctica la temática de evaluación y reducción de riesgos se incorporó a la dimensión ambiental de manera tangencial.

Ante los grandes vacíos de manejo de esta temática se optó por considerar como requisito mínimo la evaluación de las amenazas a escala general sin estudiar el contexto local, ni la vulnerabilidad y resiliencia de la comunidad. De esta manera, en el ordenamiento territorial sólo se incluyen estudios de amenaza que sirven de base para las decisiones, sin definir los grados de riesgo y su ubicación territorial, lo cual ha creado conflictos de orden social, económico, político y territorial en algunos municipios afectados permanentemente por riesgos. La falta de información lleva a tomar decisiones de organización que no corresponden con las necesidades establecidas.

A partir de esta situación, el Ministerio del Ambiente implementó una estrategia de generar procesos de capacitación con el fin de garantizar que los municipios tuvieran claridad de los temas que en materia de amenaza y riesgo debía contener un Plan de Ordenamiento Territorial; además, a través de la construcción de un instrumento denominado "Planes de Acción para la Incorporación de la Gestión del Riesgo en los POT", en el que se priorizó una ruta para mejorar su incorporación a través de procesos de revisión y ajuste o mediante la realización de estudios técnicos. Otra estrategia complementaria al ordenamiento territorial y de apoyo a los municipios ha sido el desarrollo de estudios de amenazas, vulnerabilidad y riesgos en áreas específicas de municipios y en regiones estratégicas, los cuales han permitido contar con ejercicios piloto que contribuyen a orientar los procesos de planificación local en materia de gestión del riesgo y en el mejoramiento integral de asentamientos y la consolidación de suelos de protección, entre otros (MAVDT, 2010).

Después de una década de los primeros Planes de Ordenamiento Territorial Municipal, estos deben ser replanteados, de tal modo que se asume una nueva fase de planeación territorial, en la cual se consideran algunas situaciones que permiten avanzar, como la comprensión de la importancia de la gestión de riesgos, la complejidad de los territorios y el entendimiento de la integridad de la ordenación del territorio; además, en esta segunda fase se hace necesaria una articulación del ordenamiento territorial con otras políticas de sustentabilidad, como la gestión de riesgos y la necesidad de un sistema interinstitucional que garantice una gestión armónica (Lavell, 2003). Por otra parte, en este punto se requiere mejorar la calidad técnica de los estudios de apoyo a la ordenación y consolidar el carácter democrático y participativo de la ordenación territorial (Massiris, 2009). 
A partir de las condiciones planteadas sobre las experiencias de la incorporación de la gestión de riesgos a la ordenación de los territorios municipales en Colombia, se puede decir que se ha efectuado un proceso, con una primera fase de planeación que introdujo a las instituciones gubernamentales y a las comunidades locales en un proyecto de formación y reconocimiento de los beneficios de una planeación del territorio, y particularmente la gestión de riesgos; aunque sin lograr aún sus objetivos de un desarrollo territorial sostenible con una máxima reducción de los riesgos de desastres, debido a unas condiciones sociopolíticas y económicas adversas que pueden ser modificadas en una segunda fase, caracterizada por un conocimiento de las condiciones locales, en términos territoriales, ambientales, socioeconómicos y políticoadministrativos, y con unos requerimientos de información y participación por alcanzar.

\section{Análisis de la aplicación de la Gestión de Riesgos desde la política del Ordenamiento Territorial}

Hasta hace algunas décadas, el tema de la gestión de riesgos sólo era abordado ante un desastre, a pesar de las condiciones geográficas del país, con sus altas probabilidades de amenazas como terremotos, remoción en masa, avalanchas e inundaciones. Las entidades territoriales solo actuaban ante una calamidad, sin reconocer en su jurisdicción las probabilidades de la ocurrencia de un fenómeno natural extremo. Actualmente, a través de las exigencias de los planes de ordenamiento territorial, todos los municipios del país abordaron la temática de los riesgos de desastre, y en un primer momento identificaron las amenazas y evaluaron la susceptibilidad de ocurrencia del evento natural, requiriendo un segundo proceso de análisis de exposición, vulnerabilidad y riesgo (Castrillón y Martínez, 2008). En este proyecto, muchos municipios del país recibieron gran apoyo técnico de las entidades del Sistema Nacional de Prevención y Atención de Desastres, de gran importancia para la formulación de un ordenamiento territorial coherente con la situación local.

Este proceso de conocimiento de los riesgos, generado por la política de ordenación del territorio, no fue realizado solamente por los técnicos; en muchos municipios del país la estrategia de la participación logró vincular a la comunidad con su territorio, al ser reconocida como poseedora de importante información útil para ordenar el territorio que habita. Esta estrategia también permitió establecer una comprensión de la protección de espacios amenazados y la reubicación de sus asentamientos.

Por otra parte, la implementación de los planes de ordenamiento territorial demostró la falta de conocimiento y manejo de la teoría y metodología relacionada con los riesgos de desastre; en la gran mayoría de casos, los profesionales vinculados a los proyectos no manejaban de manera adecuada los 
términos de amenaza, vulnerabilidad y riesgo; cada técnico diagnosticó los riesgos desde su formación unidisciplinar, y predominaron los estudios técnicos de las amenazas, aunque con graves problemas de clasificación por magnitudes, funcionamiento $\mathrm{y}$, sobre todo, al evaluar y dar prioridad a las amenazas presentes en el territorio; en esta situación, temas como el de la vulnerabilidad social no se trataron y en la mayoría de diagnósticos se asumió la identificación de las amenazas como la evaluación del riesgo. Desde el punto de vista territorial, los estudios de riesgos en los planes de ordenamiento tenían muchas equivocaciones en cuanto a temas como el de escala de la información y escala de ordenación, o de claridad en los elementos cartografiados, y mucho más en el manejo de técnicas cartográficas que muchas veces no cumplían los mínimos requisitos de legibilidad y ubicación, lo cual llevó a tomar decisiones poco acertadas en el momento de analizar los usos del suelo y su reglamentación.

En los planes de ordenamiento territorial se deben desarrollar diferentes fases, como el diagnóstico, la formulación, la ejecución y el seguimiento o control; cada una tiene unos requerimientos específicos y continuos. Al realizar los análisis se ha identificado una total incoherencia entre el diagnóstico de los riesgos y la formulación de proyectos relacionados con el tema, y, a su vez, entre la formulación y la ejecución, pues, al parecer, falta el conocimiento metodológico y técnico para la incorporación de la gestión del riesgo en los procesos de planificación. Al faltar esta coherencia, muchos proyectos planteados como prioritarios no se ejecutan, además, las administraciones municipales no realizan las apropiaciones presupuestales adecuadas para abordar la temática de prevención de desastres en sus jurisdicciones, debido a la incertidumbre que representan estos eventos y a que se ha hecho mayor énfasis en la atención de desastres.

\section{Conclusión}

Después de conocer el desarrollo teórico y conceptual de la gestión de riesgos y del desarrollo territorial y de revisar las políticas de ordenamiento territorial y la incorporación del tema de riesgos de desastres, se puede decir que Colombia vive un proceso en el que la gestión de riesgos y el ordenamiento territorial cuentan con unas políticas novedosas y adecuadas a sus condiciones; pero al analizar su aplicación se encuentra que, a pesar de los avances en el conocimiento local y administrativo, se dan ausencias de conocimiento teórico y metodológico, ausencias de reconocimiento de los propósitos e importancia de la gestión de riesgo y ausencia de responsabilidades y ejecuciones. Al parecer, las condiciones no están dadas aún para el logro de sus objetivos, pero lo importante para el país es que se está en un proceso hacia una gestión viable y eficiente del riesgo. 


\section{Literatura citada}

Bertrand, G. (1988). "Paisaje y geografía física global, esbozo metodológico". En: Traducciones Geográficas, pp. 11-43. Bogotá: IGAC.

Blaikie, P. et al. (1997). Vulnerabilidad del entorno social, económico y político de los desastres. Lima: FLACSO/ LA RED/ CEPREDENAC.

Calderón Aragón, G. (2001). Construcción y reconstrucción del desastre. México: Plaza y Valdés.

Cardona, O. D. et al. (2001). La necesidad de pensar de manera holística los conceptos de vulnerabilidad y riesgo. Bogotá: Centro de Estudios sobre Desastres y Riesgos -CEDERI-, Universidad de Los Andes.

Cardona, O. D. et al. (2004). "La noción de riesgo desde la perspectiva de los desastres: Marco conceptual para su gestión integral". IDEA, en: Memorias de la Conferencia Interamericana sobre Reducción del Riesgo de los Desastres. Manizales: Universidad Nacional de Colombia.

Castrillón Ojeda, C. y Martínez, M. (2008). Plan de Ordenamiento Territorial de Olaya Herrera -Nariño-. Popayán: Universidad del Cauca, Ministerio de Ambiente, Vivienda y Desarrollo Territorial.

Chías Becerril, L.; Trujillo, M. y Martínez, A. (2004). "La inseguridad vial en México: Paradigmas para su análisis geográfico". Perspectiva Geográfica 10: 145-163. Revista del Programa EPG. Bogotá: UPTC-IGAC.

Chías Becerril, L. (2009). Diagnóstico espacial de los accidentes de tránsito en el Distrito Federal. Disponible en: http://www.cenapra.salud.gob.mx/imgs/htm2/2009.

Conferencia Mundial sobre la Reducción de los Desastres. (2005). "Marco de Acción de Hyogo para 2005-2015: Aumento de la resiliencia de las naciones y las comunidades ante los desastres", Kobe, Hyogo, Japón. En: Estrategia Internacional para la Reducción de Desastres. Disponible en: www.unisdr.org.

Cuny, F. (1983). Desastres y desarrollo. New York: Oxford University.

DNP. (2001). Estrategia para consolidar la ejecución del Plan Nacional para la Prevención y Atención de Desastres. Documento Conpes 3146. Bogotá: Departamento Nacional de Planeación -DNP-.

Farinós, J. y Romero, J. (Eds.). (2007). Territorialidad y buen gobierno para el desarrollo sostenible. Nuevos principios y nuevas políticas en el espacio europeo. Barcelona: Universidad de Valencia.

296 Martha Teresa Martínez Rubiano 
GIRA -Grupo de Investigación en Riesgos Ambientales-. (2003). Estudio de vulnerabilidad social del municipio de Tumaco. Popayán: Convenio Dirección de Prevención y Atención de Desastres - Universidad del Cauca, Programa de Geografía.

Hildenbrand, A. (2007). "Tres propuestas para una relación efectiva entre las escalas regional y local en materia de ordenación del territorio". En: Farinós, J. y Romero, J. (Eds.): Territorialidad y buen gobierno para el desarrollo sostenible. Nuevos principios y nuevas políticas en el espacio europeo. Barcelona: Universidad de Valencia. pp. 147- 190.

Lavell, A. (1994). Viviendo en riesgo, comunidades vulnerables y prevención de desastres en América Latina. Bogotá: FLACSO/ LA RED / CEPREDENAC.

Lavell, A. (2003). La gestión local del riesgo. Nociones y precisiones en torno al concepto y la práctica. CEPREDENAC-PNUD.

Martínez R., M. (2005). Tumaco: comunidad vulnerable ante tsunami. Popayán: Editorial Universidad del Cauca.

Martínez R., M. (2007). "Perspectiva geográfica de la vulnerabilidad ante amenazas de origen natural". En: Memorias del XI Encuentro de Geógrafos de América Latina (EGAL), Geopolítica, globalización y cambio ambiental, retos en el desarrollo latinoamericano, pp. 121-122. Bogotá: Universidad Nacional.

Maskrey, A. (1989). El manejo popular de los desastres naturales. Estudios de vulnerabilidad y mitigación. Lima: ITDG.

Massiris, A. (2000). "El diagnóstico territorial en la formulación de planes de ordenamiento". Perspectiva Geográfica, 5: 33-54. Revista del Programa de Estudios de Posgrado en Geografía. Bogotá: Uptc-IGAC.

Massiris, A. (2009). "Desarrollo territorial sostenible en América Latina". en: Farinós J., Romero J. y Salom, J. (Eds.): Cohesión e inteligencia territorial, Dinámicas y procesos para una mejor planificación y toma de decisiones. Barcelona: Universidad de Valencia. p. 121-138.

MAVDT. (2006). La gestión de riesgos, un tema de Ordenamiento Territorial. Ruta para la toma de decisiones. Serie Ambiente y Ordenamiento Territorial. Bogotá: Ministerio de Ambiente, Vivienda y Desarrollo Territorial.

MAVDT, SNPAD, DNP y Acción Social. (2005). Guía Metodológica 1, Incorporación de la prevención y la reducción de riesgos en los procesos de Ordenamiento Territorial. Serie Ambiente y Ordenamiento Territorial. Bogotá: Ministerio de Ambiente, Vivienda y Desarrollo Territorial.

MAVDT, SNPAD, DNP y Acción Social. (2006). Guía para orientar las acciones $e$ inversiones en gestión local del riesgo a nivel municipal. Bogotá: Ministerio de Ambiente, Vivienda y Desarrollo Territorial.

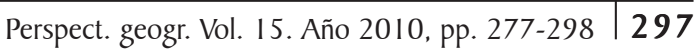


MAVDT, ESAP, ONU-HABITAT, PNUD. (2004). Formulación y aplicación de la ley 388 de 1997 en Colombia - Una práctica colectiva hecha realidad-, Políticas urbanas y legislación facilitadora. Nairobi: ONU-HABITAT, PNUD.

Santos, M. (2000). La naturaleza del espacio. Barcelona: Ariel.

Thomas Castellanos, M. (2006). Memorias del Diplomado en Gestión de Riesgos. Popayán: Grupo de Investigación en Riesgos Ambientales, Universidad del Cauca.

Fecha de recepción: 25 de octubre de 2010

Fecha de aprobación: 6 de diciembre de 2010

298 Martha Teresa Martínez Rubiano 